\title{
Editorial
}

Digestion

\section{Commentary Regarding Nighttime Reflux}

\author{
Lars Lundell \\ Department of Surgery, Gastrocentrum, Karolinska University Hospital, Huddinge, Stockholm, Sweden
}

With the expanding knowledge on the key pathogenetic mechanisms behind gastroesophageal reflux disease (GERD), in which it has become clear that neurogenic control of the lower esophageal sphincter (LES), supported by the function of the cural diaphragm, is provital, the management focus for these patients has been directed toward control of daytime reflux.

This model is based mainly on the role of transient LES relaxations triggered by the excitation of tensionmechanoreceptors located in the area of the proximal stomach and gastric cardia. These transient LES relaxations are the main mechanisms behind daytime reflux and occur predominantly in the postprandial period. As a result of this, daytime reflux has traditionally been well recognized by clinicians involved in the treatment of the large group of patients suffering from GERD. This is particularly the case in the majority of GERD patients, i.e. those who have non-erosive GERD. This latter group has repeatedly been shown to suffer from isolated daytime reflux. Despite these findings recent clinical research has revealed that quite a significant proportion of these patients suffer from sleep disturbances. Although the pathophysiological mechanisms behind similar nighttime-related complaints have to be clarified, it is intriguing to note that profound acid inhibition therapy has dramatic effects. Corresponding data enable a novel approach to the recognition of reflux occurring in the supine position, e.g. that occurring close to the gastroesophageal junction, which traditionally has not been picked up by conventional ambulatory $\mathrm{pH}$ monitoring.

A substantial amount of data has been gathered on nighttime reflux in patients with severe manifestations of reflux disease, i.e. those with LA grade $\mathrm{C}$ and $\mathrm{D}$ as well as in those with Barrett's esophagus. In many of these cases a single mechanical explanatory model of the gas- troesophageal junction has offered pathogenetic and therapeutic guidance. As an extension to a similar mechanical deficiency approach, surgical reconstruction has frequently been recommended in patients in whom medical therapy fails, and even earlier in the decision-making process relevant to clinical management. Isolated nighttime reflux is an uncommon phenomenon. On the other hand it is regularly seen in complicated reflux disease. The great problem with reflux during the night is not only the apparent consequences on esophageal clearance that the supine body position has, but also the frequent prevalence of severe esophageal motor disturbances in similar patients. Moreover, the pharmacological and pharmacokinetic properties of proton pump inhibitors are such that acid reaction regularly breaks through during the late night hours. This may in many situations reflect a potential advantage allowing, for instance, the bactericide effect of acid to partly clear the stomach from bacterial overgrowth. The understanding of these properties is, however, crucial to adequately treat patients with complicated reflux disease in general and those with nighttime reflux in particular. In routine clinical practice these issues have not received enough attention which is why the current review article plays a particular role.

Furthermore, quite complicated therapeutic regimens with proton pump inhibitors combined with H2RA have been launched. For obvious reasons many clinicians have not been too enthusiastic about such complex pharmacotherapeutic approaches. Also, based on these aspects, the current review offers guidance to clinicians to adequately select the right drug and appropriate dosing to reach valid therapeutic goals. Finally the comprehensive collection and compilation of data completed by Dr. Orr also define areas for future research based on the definition of unmet needs in the management of patients with GERD.

\section{KARGER \\ Fax +4161306 1234 E-Mail karger@karger.ch}

www.karger.com
(C) 2005 S. Karger AG, Basel 0012-2823/05/0724-0228\$22.00/0

Accessible online at: www.karger.com/dig
Lars Lundell, $\mathrm{MD}, \mathrm{PhD}$

Department of Surgery, Gastrocentrum

Karolinska University Hospital, Huddinge

S-141 86 Stockholm (Sweden)

E-Mail lars.lundell@karolinska.se 